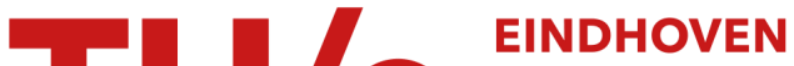 UNIVERSITY OF TECHNOLOGY
}

\section{Experimental and theoretical study of semiconductor laser dynamics due to filtered optical feedback}

\section{Citation for published version (APA):}

Fischer, A. P. A., Yousefi, M., Lenstra, D., Carter, M. W., \& Vemuri, G. (2004). Experimental and theoretical study of semiconductor laser dynamics due to filtered optical feedback. IEEE Journal of Selected Topics in Quantum Electronics, 10(5), 944-954. https://doi.org/10.1109/JSTQE.2004.835997

DOI:

10.1109/JSTQE.2004.835997

Document status and date:

Published: 01/01/2004

\section{Document Version:}

Publisher's PDF, also known as Version of Record (includes final page, issue and volume numbers)

\section{Please check the document version of this publication:}

- A submitted manuscript is the version of the article upon submission and before peer-review. There can be important differences between the submitted version and the official published version of record. People interested in the research are advised to contact the author for the final version of the publication, or visit the $\mathrm{DOI}$ to the publisher's website.

- The final author version and the galley proof are versions of the publication after peer review.

- The final published version features the final layout of the paper including the volume, issue and page numbers.

Link to publication

\section{General rights}

Copyright and moral rights for the publications made accessible in the public portal are retained by the authors and/or other copyright owners and it is a condition of accessing publications that users recognise and abide by the legal requirements associated with these rights.

- Users may download and print one copy of any publication from the public portal for the purpose of private study or research.

- You may not further distribute the material or use it for any profit-making activity or commercial gain

- You may freely distribute the URL identifying the publication in the public portal.

If the publication is distributed under the terms of Article 25fa of the Dutch Copyright Act, indicated by the "Taverne" license above, please follow below link for the End User Agreement:

www.tue.nl/taverne

Take down policy

If you believe that this document breaches copyright please contact us at:

openaccess@tue.nl

providing details and we will investigate your claim. 


\title{
Experimental and Theoretical Study of Semiconductor Laser Dynamics Due to Filtered Optical Feedback
}

\author{
A. P. A. Fischer, Mirvais Yousefi, Member, IEEE, D. Lenstra, Michael W. Carter, and Gautam Vemuri
}

\begin{abstract}
We report experimental results on the nonlinear dynamical response of a semiconductor laser subjected to time-delayed $(>5 \mathrm{~ns})$, frequency selective, optical feedback from a Fabry-Pérot interferometer type of filter. Three regimes of interest, based on the relative value of the filter bandwidth with respect to the relevant laser parameters (relaxation oscillation frequency and external cavity mode spacing), are identified, viz. a wide filter case, an intermediate filter width case, and a narrow filter case. The dynamical response of the laser is shown to be quite different in each of these regimes. The principal results are 1) the laser's linewidth enhancement factor, coupled with the nonlinear response of the filter, can be exploited to induce nonlinear dynamics in the instantaneous optical frequency of the laser light on a time scale related to the time-delay of the feedback, 2) a mode mismatch effect which arises from a detuning between the filter center frequency and the nearest external cavity mode and manifests itself in a reduction of the maximum light available for feedback, and 3) a reduction in, or even disappearance of, relaxation oscillations in the laser dynamics when a filter of appropriate width is chosen. More generally, it is observed that certain dynamics that occur due to unfiltered optical feedback may be suppressed when the feedback light is spectrally filtered.
\end{abstract}

Index Terms-Diode lasers, filtered feedback, frequency dynamics, mode-mismatch, optical feedback, time-delay.

\section{INTRODUCTION}

$\mathbf{T}$ HE EXTREME sensitivity of semiconductor lasers to optical injection and to time-delayed feedback, whether electronic or optical, has been thoroughly studied in recent years to understand why the performances of these lasers is sometimes improved, while at other times degraded, by these perturbations. Also, the nonlinear dynamical response of a semiconductor laser subject to optical feedback has received tremendous attention [1]. Lasers subject to time-delayed feedback are a paradigm for fundamental investigations on the response of systems subject

Manuscript received January 26, 2004; revised July 27, 2004. This work was supported by the European Community under Contract HPRI-CT-1999-00064. The work of G. Vemuri and M. W. Carter was supported by the National Science Foundation. The work of D. Lenstra and M. Yousefi was supported by the Belgian IAP-18 Network "PHOTON."

A. P. A. Fischer is with the Laboratoire de Physique des Lasers, Université Paris XIII, UMR CNRS 7538, 93430 Villetaneuse, France.

M. Yousefi was with the Vrije Universiteit Amsterdam, $1081 \mathrm{HV}$, Amsterdam, The Netherlands. He is now with the Vrije Universiteit Brussels, B-1050 Brussels, Belgium and also with the COBRA Research Institute, Technical University, 5600 MB Eindhoven, The Netherlands (e-mail: myousefi@tona.vub.ac.be).

D. Lenstra is with the Vrije Universiteit Amsterdam, $1081 \mathrm{HV}$, Amsterdam, The Netherlands and also with the COBRA Research Institute, Technical University, 5600 MB Eindhoven, The Netherlands (e-mail: lenstra@nat.vu.nl).

M. W. Carter and G. Vemuri are with the Department of Physics, Indiana University-Purdue University, Indianapolis, IN 46202-3273 USA (e-mail: gvemuri@iupui.edu).

Digital Object Identifier 10.1109/JSTQE.2004.835997 to delayed perturbation, and, hence, this has become a topic of substantial interest in physics and mathematics [2]. From the standpoint of the mathematical formalism, the rate equations that describe lasers subject to delayed feedback have the form of delay differential equations, where a critical variable, such as the laser electric field, is a function of its time-delayed replica. Such equations then become infinite-dimensional because to calculate a physical quantity, say the electric field, at a given instant of time, one has to specify the history of that quantity over a period of one delay time prior to that. This can make analytic solutions to the governing equations a formidable challenge, and yet, this infinite dimensional nature of the problem is precisely the reason that the laser subject to feedback exhibits many interesting dynamical behaviors [3]-[6].

Many of the early studies on semiconductor lasers driven by delayed feedback were directed toward an elaboration of the various possible dynamics, and an elucidation of their origin and the parameters that govern them. However, lately the focus has shifted toward a utilization of the dynamical behaviors for applications such as high-speed optical communications and cryptography [7]. This has led to a realization that in order to profitably employ the dynamics in appropriate devices, one must develop robust techniques that enable one to control the dynamical response of the laser. Filtered optical feedback(FOF), wherein the feedback is spectrally filtered before it is fed back into the laser, is one possible candidate for exercising this control, as we have shown in recent papers [8]-[10]. Of course, spectral filtering of feedback light into a semiconductor laser is routinely found in certain applications where the laser dynamics are not a relevant issue.

For example, using feedback from a diffraction grating is a common method for obtaining single-mode operation of a semiconductor laser. In such cases, a specific spectral component of the diffracted light is fed back to the laser, and hence this forms a good example of utilizing filtered feedback. Recent work has also shown the utility of a Michelson interferometer to affect the low frequency fluctuations (LFF) related intensity dropouts seen in a semiconductor laser subject to optical feedback [11], [12]. This is another example of using filtered feedback to control the behavior of the laser. In other applications, an alkali vapor or a filter is placed within the external cavity laser system to frequency stabilize the laser. In such configurations, the laser frequency is locked to one of the transition lines of e.g., Rubidium or Barium, or to a resonance of a Fabry-Pérot interferometer. All of these systems are equivalent to a semiconductor laser with an external cavity containing a frequency-selective filter.

Unlike conventional optical feedback (COF), where the frequency content of the feedback light is unaltered, employing a filter immediately provides two parameters, that are external 
to the laser, to control the laser dynamics. These are the bandwidth of the filter, and its detuning from the solitary laser frequency (i.e., in the absence of any feedback). A manipulation of these two parameters in FOF leads to a variety of interesting dynamics. For example, we demonstrated recently, through experiments and numerical modeling, that via a judicious choice of the filter bandwidth, and its detuning from the laser, one can induce dynamics in the instantaneous optical frequency of light from the laser, and that one can ensure that the time scale of the dynamics is dominantly set by the time-delay of the feedback light [13]. Dynamics that arise during COF are usually both in the intensity and the frequency of the laser light, and the time scale is dominated by the relaxation oscillation frequency $\left(\nu_{R O}\right)$. During FOF, one can select the filter bandwidth, $\delta \nu_{f}$, to be much narrower than the $\nu_{R O}$ and carefully set other parameters so as to filter out the relaxation oscillations. This permits one to produce controlled dynamics in the laser, on a time scale that is determined through a choice of the delay.

The key difference between COF and FOF dynamics arises from the fact that in the latter case one can exploit the cooperation between the linewidth enhancement factor of the laser (also called the $\alpha$ parameter) and the nonlinear frequency profile of the filter. This can be qualitatively understood by referring to Fig. 1. It is well known that the $\alpha$-parameter causes the frequency of the laser to red shift by an amount that depends on the amount of the feedback light. Suppose the filter is detuned from the solitary laser such that the filter transmission function has the value $T_{o}$ at the laser frequency. The light coming through the filter will have the same frequency as before, but will have an amplitude of $T_{o} \varepsilon$. On injection into the laser, this light will red-shift the laser frequency by an amount $\delta \omega$ that depends on $\alpha$, such that the laser frequency is now $\omega^{\prime}$ instead of the original $\omega$. Now, this light, of frequency $\omega^{\prime}$, will be incident on the filter and produce a different transmission value, thereby resulting in a different amplitude of feedback light. Again, when this light enters the laser, it will lead to a new frequency that is in principle different from $\omega^{\prime}$. Thus, one can imagine that it is possible to loop the filter and the laser in such a way that one produces controlled excursions in the frequency of the laser light.

Recently, one of us showed that the effect of a filter is to select one or a group of external cavity modes to which the laser locks, thus allowing a better control of its stability or switching time. Specifically, with such an optical feedback device, one can achieve a faster and more accurate wavelength switching than what is obtained by simply changing the pump current, as is usually done [14]. Sweeping the pump current is accompanied by thermal fluctuations which are very slow $(\sim \mathrm{kHz})$, and so varying the feedback may be a better method. Indeed fast switching devices would be useful not only in wavelength division multiplexing (WDM)-systems but also for signal-routing. Moreover the selection of a group of external cavity modes might become interesting for hyper-dense WDM.

There are a few instances in the literature where the effect of FOF in semiconductor lasers on the laser dynamics has been the subject of study. Huyet et al. considered the low-frequency fluctuations dynamics, and their possible origin, through experiments where a diffraction grating was used to selectively filter the frequency of the feedback light [15], [16]. Goedgebuer et
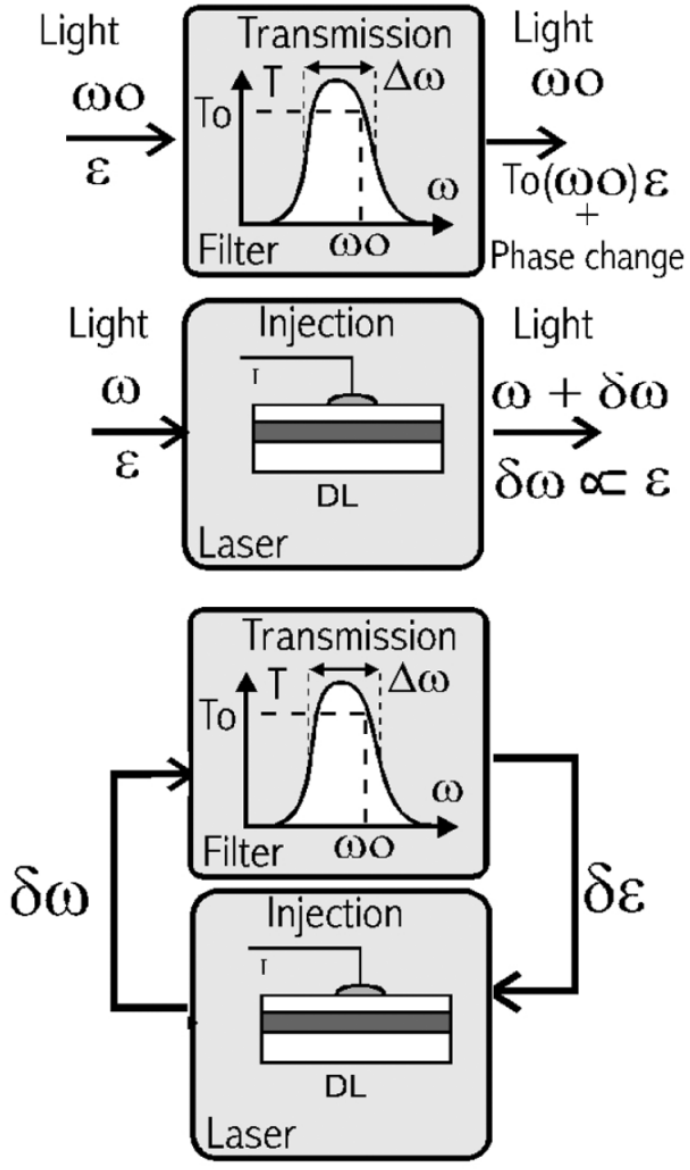

Fig. 1. Schematic to motivate the origin of dynamics in the frequency of light from a semiconductor laser subject to FOF. If light of frequency $\omega_{0}$ and amplitude $\varepsilon$ is incident on a filter at a frequency where the transmission function of the filter has a value $T_{o}$, then the light transmitted by the filter has an amplitude $T_{o} \varepsilon$. If light of frequency $\omega$ and amplitude $\varepsilon$ is incident on a semiconductor laser, then due to the $\alpha$-parameter of the laser, the transmitted light is red-shifted by an amount proportional to $\varepsilon$. Coupling the laser and the filter together can then result in excursions in the instantaneous optical frequency, as described in the text.

al. have considered FOF in the context of optoelectronic feedback and demonstrated frequency dynamics, as well as chaotic synchronization of two such lasers [17], [18]. Our emphasis, in this paper, is on all-optical feedback, with special attention to the aspects of dynamics that arise from such feedback. The principal differences arise from the fact that in our experiments the feedback is coherent, whereas in the case of optoelectronic feedback it is incoherent. It is known that two important factors determining the dynamical behavior of the laser are the amount of feedback and, in the case of coherent feedback, the phase difference between the emitted wave and the perturbing, feedback wave. The practical implication of the latter is that one obtains a set of external cavity modes (ECMs) in the case of coherent feedback, whereas ECMs are not meaningful in case of incoherent feedback. The number of ECMs within the filter profile determines the complexity of the dynamics, and so controlling that number is one instrument to force the laser into desired dynamical regimes [19].

The main purpose of this paper is to demonstrate the principles behind FOF that affect the dynamical behavior of a semiconductor laser. In particular, we identify three regimes of in- 
terest, based on the relative values of the filter bandwidth, the external cavity mode spacing $\left(\Delta \nu_{\mathrm{ECM}}\right)$, and the relaxation oscillation frequency $\left(\nu_{R O}\right)$ of the laser. We show that the laser's dynamical response is substantially different in each of these three regimes, and pay special attention to the following aspects.

1) The filtering property of the filter, where its width relative to the other parameters influences the course of the dynamics. For example, a very narrow filter may suppress relaxation oscillations, but will also permit very few ECMs within the filter profile, thereby generally leading to very simple dynamics, whereas a very broad filter will support a large number of ECMs but it will also allow the relaxation oscillations to leak in. An intermediate filter width, such that it blocks the relaxation oscillations, and yet permits a number of ECMs under the filter to produce interesting dynamics, probably is the most interesting from a dynamical perspective.

2) Mode mismatch: An effect that can occur when the filter center frequency moves through the ECM frequencies. In particular, if the solitary laser frequency is not coinciding with an ECM frequency, one can get a substantial reduction in the feedback light (this will normally be the case, except for special external mirror distance settings). This effect is a unique feature of coherent feedback, and as we will show, can have a substantial influence on the amplitude of the feedback light and thereby affect the dynamics.

3) The occurrence of dynamical behaviors in the frequency of the laser light on a time scale that is predominantly set by the delay time of the optical feedback. We emphasize that the frequency dynamics we discuss here are a qualitatively new type of dynamics that are not observed during COF. During COF, the dynamics arise from an undamping of the relaxation oscillation frequency and one observes dynamical behaviors in the intensity and frequency of the laser light. In contrast, during FOF, when the parameters are chosen to preclude any undamping of the relaxation oscillations, we observe dynamics in the frequency only, and the intensity of the light is quite stable. These three effects will appear on different occasions when the filter linewidth inside the external cavity is varied.

\section{DESCRIPTION OF THE EXPERIMENTAL SETUP}

A schematic of the experiment is shown in Fig. 2. It consists of a semiconductor diode laser (DL) with an external-cavity loop, which includes the spectral filter $(F)$, and a diagnostic branch. The DL is a commercial, single-mode, 5-mW FabryPérot type semiconductor laser (SHARP LT027) emitting at 780 $\mathrm{nm}$, with a threshold current of $46 \mathrm{~mA}$ (free running) and operated in the range 55-75 mA. The external-cavity loop consists of a neutral-density filter, a beam splitter BS1, and the spectral filter F. This filter F consists of two mirrors M1 and M2 resulting in a finesse $f$, and spaced by the distance $d$, while the distance between the DL and M1 is $L$. The first mirror M1 is wedged so as to eliminate the reflection from its first facet which produces a pass band transfer function in reflection.

The diagnostic branch consists of five arms (A, B, C, D, and E) isolated from the rest of the setup by three optical isolators

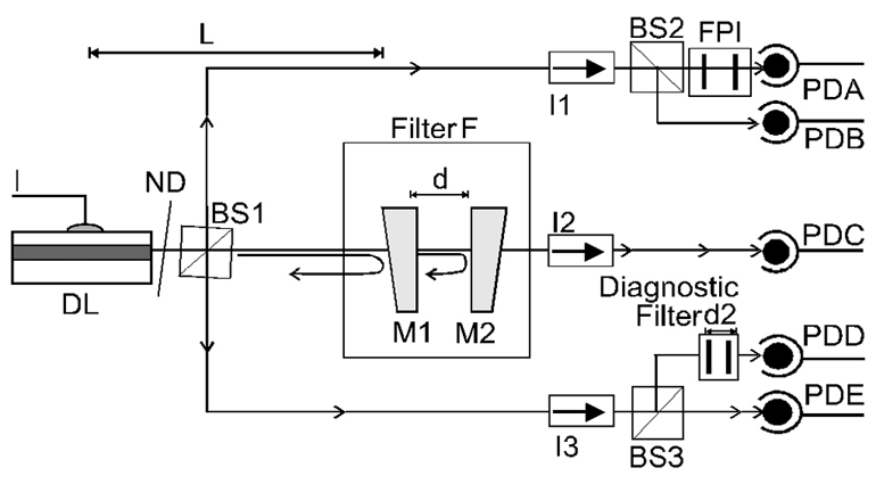

Fig. 2. Schematic of the experiment for observation of frequency dynamics. DL: Diode laser. ND: tunable neutral density filter. M1, M2: mirrors of the filter. I1, I2, I3: optical isolators. BS1, BS2, BS3: beam splitters. PDA, PDB, PDC, PDD, PDE: photodiodes. FPI: Fabry-Pérot interferometer.

(I1 I2, and I3) and each arm terminates with a photodiode PDA, PDB, PDC, PDD, and PDE, respectively. I1 and I3 prevent parasitic reflections from the arms $\mathrm{A}, \mathrm{B}, \mathrm{D}$, and $\mathrm{E}$ through $\mathrm{BS} 1$, whereas $\mathrm{I} 2$ shields from parasitic reflection from arm $\mathrm{C}$ through the filter and the mirrors M1 and M2. A portion of the light emitted by the DL is split-off by BS1 and directed into arms A and B. Arm A consists of a scanning 250-MHz FSR Fabry-Pérot interferometer (FPI) with a finesse of 25 and a photodiode PDA, and is used to obtain the optical spectrum of the laser light with a resolution of $10 \mathrm{MHz}$. Arm B has a 1-GHz bandwidth photodiode PDB with a 30-dB amplifier, and measures the optical power in the laser light. Arm $\mathrm{C}$ contains a 1-GHz bandwidth photodiode PDC located behind the filter F. Arms D and E monitor the feedback light. In arm E, PDE detects the feedback signal, the DC part of which gives the average feedback level, while the AC part contains the instantaneous power variations. Lastly, in arm D, a diagnostic filter is located before the 1-GHz photodiode PDD. The diagnostic filter is a Fabry-Perot type of interferometer with a spacing $d 2$ between the mirrors, and it permits a measurement of the dynamics in the frequency of the laser light since it converts the instantaneous frequency excursions into power variations. Clearly, the bandwidth of this diagnostic filter is an important parameter; the width has to be large enough to permit both a linear response and a short response time, and yet sharp enough to produce measurable power variations. In practice, this filter width was typically two to five times the width of the spectral filter $(F)$ that was used to filter the feedback light. The filter F located before the photodiode PDC plays a role similar to the diagnostic filter for PDD, and in practice the signals observed on PDC offer a better contrast than those measured on PDD.

The path of the light in the external cavity is as follows: light emitted by the DL propagates through ND to BS1. Both elements are misaligned slightly so as to avoid spurious feedback from their surfaces, and care was taken to ensure that the total parasitic feedback was much below $-55 \mathrm{~dB}$ and, hence, had no influence on the DL dynamics. Part of the light was split off and used for diagnostics in arms A, B, D, and E, and the rest of the light entered the Fabry-Pérot filter F (M1 and M2). M1 was a 3-mm-thick, wedged mirror so that no multiple reflections occur inside the mirror M1, and such that the reflection from the first facet facing the DL is eliminated from the filter transfer function 
resulting in a pass band filter profile. The transmitted light then encountered a similar wedge mirror M2, spaced by a distance $d$ from M1. Both mirrors were fixed on accurate, fine tuning mechanical mounts so that M2 could be aligned to reflect the light directly into the DL. Depending on the alignement of M2 with respect to M1 the finesse $f$ ranges between $f=3$ and $f=10$. When M2 was slightly misaligned, multiple reflections between M1 and M2 did not coincide, and one could thus obtain an approximate measure of the finesse $f$ of the filter $\mathrm{F}$ by counting the number of reflected spots. Multiple pass interference was built up by aligning M2 so that all the spots overlap onto the front facet of the DL. In this manner, the filtered optical feedback situation was achieved.

After realignment of M2, the behavior of the DL was analyzed through the diagnostic part of the setup. Since F is a Fabry-Pérot interferometer, a frequency selection takes place and both the amount of light fed back and transmitted depend on the DL frequency. The transmission is monitored in arm C. When the total feedback is kept below $-55 \mathrm{~dB}$ and the injection current to the DL scanned (thereby scanning its frequency), one obtains the transmission and reflection transfer curves of the filter on PDC and PDE, respectively. The spectral properties of the filter, such as the linewidth, and the free spectral range, can thus be measured. A similar setup but with a looped external cavity structure has also been studied and produces similar results [9].

\section{OUTLINE OF STUDY}

The laser with FOF system is characterized by four important time scales. The first is the relaxation oscillation (RO) frequency, $\nu_{R O}$ of the DL [19], which is a critical parameter in both $\mathrm{COF}$ and FOF. It is in good approximation given by

$$
v_{R O}=\frac{1}{2 \pi} \sqrt{\xi \Gamma_{o} P}
$$

where $\xi$ is the differential gain, $\Gamma_{0}$ is the photon decay rate, and $P$ the number of photons in the DL, and has a value of the order of 4-8 GHz.

The second time scale follows from the ECM-free spectral range $\Delta \nu_{\mathrm{ECM}}=c / 2 L$, where $L$ is the spacing between the laser and the external mirror from which the reflection of light occurs. The ECMs arise due to coherent feedback, as mentioned earlier, and the ECM frequency can be varied by changing the external cavity length. In the cases studied here, $\Delta_{\mathrm{ECM}}<\sim$ $200 \mathrm{MHz}$, corresponding to typically long external path length $L>0.75 \mathrm{~m}$.

The presence of the filter introduces two more time scales, the filter bandwidth [full-width at half-maximum (FWHM)] $\delta \nu_{f}=c / 2 f d$, where $f$ is the finesse, and the filter free spectral range (FSR) $\Delta \nu_{f}=c / 2 d$. These frequencies are the easiest controllable external parameters by simply changing the mirror spacing. In our experiments, the FWHM $\delta \nu_{f}$ can be set from few megahertz up to few gigahertz and covers all the different time scale ranges. On the basis of these frequencies, we can distinguish three cases depending on the value of filter bandwidth $\delta \nu_{f}$ relative to the RO frequency $\nu_{R O}$ and the ECM spacing
$\Delta \nu_{\mathrm{ECM}}$. Each case leads to different dynamical behaviors that will be investigated in the rest of the paper.

1) Wide Filter Case: $\delta \nu_{f}>\nu_{R O}$

The filter bandwidth is larger than the RO frequency so that when the filter is centered at the solitary laser frequency, the RO-side-peak falls within the filter profile. This case is very close to COF, wherein, one can have several hundred external cavity modes under the filter profile, and so the dynamics can be quite complicated.

2) Intermediate Filter Case: $\Delta \nu_{\mathrm{ECM}}<\delta \nu_{f}<\nu_{R O}$

The filter width is smaller than the RO frequency but larger than the external cavity mode spacing. One can have a few tens of ECMs under the filter profile, such that the dynamics are more complicated than the narrow filter case, but in this regime one has the possibility to control dynamics.

3) Narrow Filter Case: $\delta \nu_{f} \ll \nu_{R O}$ and $\delta \nu_{f}<\Delta \nu_{\mathrm{ECM}}$ In this case, the filter is so narrow that at most one ECM lies under the filter profile and so the laser prefers to operate on that single external cavity mode. Yet even here dynamics are possible. One mechanism for dynamics is the destabilization of the ECM through a Hopf bifurcation, leading to undamped ROs. Another mechanism is to detune the solitary laser by one RO frequency with respect to the filter center. The feedback channel via the RO side peak will likely give rise to undamped ROs. In fact, these two mechanisms are also active in the intermediate case.

In Section IV, we present, for the three cases mentioned, the results of experimental measurements and numerical simulations, on semiconductor laser dynamics due to FOF. The experimental results were obtained with the setup described in Section II. The simulations utlize a rate equation model for filtered optical feedback that has been described in detail in [19], and so will be briefly reviewed here: the optical field in the laser is represented by $E(t)=E(t) \exp \left\{i \omega_{0} t\right\}+c . c$. , where $\omega_{0}$ is the operation frequency of the laser in the absence of feedback (to be referred to as the "solitary laser"), while $E(t)$ is the (complex) slowly varying amplitude. The model equations read

$$
\begin{aligned}
\dot{E}(t) & =\frac{1}{2}(1+i \alpha) \xi n(t)+\gamma F(t) \\
\dot{F}(t) & =\Lambda E(t-\tau) \exp \left(-\omega_{0} \tau\right)+\left(i \omega_{f}-\Lambda\right) F(t) \\
\dot{n}(t) & =J-J_{t h r}-\frac{n(t)}{T_{1}}-\left[\Gamma_{0}+\xi n(t)\right]|E(t)|^{2} .
\end{aligned}
$$

Here, $F(t)$ is the (complex) field amplitude re-entering from the laser cavity, $n(t)$ describes the inversion, or the number of electron-hole pairs relative to their value at solitary laser operation, $\Lambda$ is the half-width at half-maximum (HWHM) of the (Lorentzian) filter, $\omega_{f}$ the center frequency of the filter relative to the solitary laser frequency $\omega_{o} . J=I / e$ is the normalized pump rate, $I$ is the pump current, $J_{t h r}$ is the pump rate at the solitary laser threshold of the specific longitudinal mode, $\omega_{t h r}$ is the corresponding threshold frequency, and $\tau$ is the external cavity round trip time.

The description of the solitary laser, according to (1) and (3), yields total clamping of the inversion above the laser threshold and, thus, implies clamping of the solitary laser frequency $\omega_{O}$. 
In a real laser, however, the clamping is not total, as a result of heating and the presence of spontaneous emission. To account for this variation we set

$$
\omega_{0}=\omega_{t h r}-k\left(J-J_{t h r}\right),
$$

where $k>0$ is an empirical constant of proportionality. In principle, since the gain coefficient $\xi$ depends on frequency, it will show variations with the pump rate as well. However, as we do not expect any significant effect resulting from the modest frequency variation range here considered $(20 \mathrm{GHz})$, the gain coefficient is taken constant. All other parameters are identified in $[19$, Table I]. The details of the numerical simulation techniques are provided therein. For each of the three cases (wide, intermediate, and narrow filter case) the simulations calculate the actual instantaneous frequency $\omega$ of the FOF system as a function of the solitary laser frequency, $\omega_{0}$, which is one of the control parameters for our system. Note that the solitary laser frequency can be controlled in the experiments through a variation of the injection current, and this dependence of $\omega_{0}$ on the current is included in the numerical model. To begin with, the fixed point solutions of the rate equations were calculated for the FOF system. They represent the continuous wave (CW)-states of operation with a monochromatic field and constant inversion. However, in many cases, the steady-state operation is not a stable solution and, therefore, it is essential to also calculate the dynamics. So next, taking a fixed point value as the initial condition, the time-averaged operating points between the mean values were obtained by integration of (1)-(3).

\section{RESULTS}

\section{A. Wide Filter Case $\left(\delta \nu_{f}>V_{R O}\right)$}

For this case, the parameters in the experiment were $L=$ $1.5 \mathrm{~m}$ for the external cavity length, a spacing of $d=2 \mathrm{~mm}$ between $\mathrm{M} 1$ and M2, and a finesse $f=10$. This results in an ECM spacing $\Delta \nu_{\mathrm{ECM}}=100 \mathrm{MHz}$ and $\delta \nu_{f}=7,5-\mathrm{GHz}$ filter width (FWHM). For these parameter values, there are about 75 ECMs present within the filter profile. When our experimental setup was optimized for maximum filtered feedback, we measured a maximum feedback power of $18.5 \mu \mathrm{W}$ whereas the filter was observed to deliver $20 \mu \mathrm{W}$ at its center frequency in the absence of feedback. This difference is slightly more than the uncertainties in the power measurements, though the actual values depend very much on the external cavity length $L$ and the mirror spacing of the filter $d$. A similar behavior, i.e., a decrease in available feedback power, was observed earlier in the first two articles mentioned in [5].

Fig. 3, shows the optical spectrum of the laser as observed with a scanning FPI, and summarizes the response of the laser when subject to different levels of feedback. In Fig. 3, the laser frequency is resonant with the filter center frequency. In the absence of feedback, one gets the spectrum of the free-running laser and the measured linewidth of the laser is about $50 \mathrm{MHz}$ [Fig. 3(a)]. When the feedback power is about $3 \mu \mathrm{W}(-32 \mathrm{~dB})$, one sees linewidth narrowing as the laser phase locks to the phase of the feedback field [Fig. 3(b)]. With increasing feedback, about $7 \mu \mathrm{W}(-29 \mathrm{~dB})$, there is an undamping of the relaxation oscillations that appear as two satellite peaks [Fig. 3(c)], which ultimately leads to coherence collapse at the maximum feedback level of $18 \mu \mathrm{W}(-25 \mathrm{~dB})$ [Fig. 3(d)]. Note that the FSR of the diagnostic Fabry-Perot used for observing the DL spectrum is $200 \mathrm{MHz}$ and the 4-GHz RO appear as a result of a back convolution. Clearly, a FOF system where the filter width is wider than the RO frequency is in many respects very similar to the COF case. In both cases, one finds that with increasing feedback there is an undamping of the relaxation oscillations, and that the dynamics of the laser are dominated by the relaxation oscillation time scale.

To elaborate upon the decrease in the maximum feedback power available, Fig. 3(e) displays the calculated frequency $\omega$ of the system relative to its value at threshold as a function of the solitary laser frequency $\omega_{0}$ (also relative to threshold). The amount of feedback light was chosen to be $18 \mu \mathrm{W}(-25 \mathrm{~dB})$, which we estimate, for use in the numerical model, to correspond to a feedback rate $\gamma=31.86 \mathrm{~ns}^{-1}$. If the solitary laser frequency $\omega_{0}$ is increased starting at a point well below the filter center frequency, the operating point moves from A along a mode (the laser locks to that mode) until it reaches a saddle node $\mathrm{B}$ where it jumps to the immediate adjacent mode $\mathrm{C}$, and locks to it until it reaches a new Saddle node D. Ultimately, it reaches the top of the filter $(\mathrm{H})$ where a jump occurs to the other side of the filter (I) and continues the same process of locking and jumping ibetween adjacent ECMs from $\mathrm{J}$ to $\mathrm{Q}$. For a complete description the reader is asked to see [9]. Note that point $\mathrm{H}$ does not necessarily lie at the top of the filter profile ( $H_{\max }$ at $\omega_{o}=\omega_{\text {cent }}=2 \pi \nu_{\text {cent }}$ ). In an experiment, this would imply that the maximum feedback power, i.e., the net amount of light coupled back into the laser, will be smaller than the power available from the filter when measured at the center of the filter in absence of feedback. We refer to the mechanism responsible for this decrease in the feedback power as the mode mismatch effect. It arises in filtered, coherent feedback due to a mismatch between the center frequency of the filter and the nearest ECM frequency.

\section{B. Intermediate Filter Case $\left(\delta \nu_{\mathrm{ECM}}<\delta \nu_{f}<\nu_{R O}\right)$}

The external cavity length was varied between $L=1.5 \mathrm{~m}$ to $5.6 \mathrm{~m}$, resulting in an ECM spacing of $100-26 \mathrm{MHz}$. The spacing between the filter mirrors was $d=2.1 \mathrm{~cm}$, and the filter had a finesse $f=10$, leading to a filter bandwidth (FWHM) $\delta \nu_{f}=700 \mathrm{MHz}$. For an $L$ of $1.5 \mathrm{~m}$, there were about $7 \mathrm{ECMs}$ present within the filter profile. In the experiments, we measured the optical power through the filter, in the absence of feedback, as $20 \mu \mathrm{W}$, while the maximum transmitted power in the presence of feedback, at the central filter frequency, was $7 \mu \mathrm{W}$. These measurements were made by slightly misaligning the external cavity so that the feedback spot is removed from the laser facet. There is then no interference between the output field of the laser and the incoming field, which allows a measurement of the dc component of the optical power (measured to be 20 $\mu \mathrm{W}$ ). In the presence of feedback, the beam-splitter BS1 is used to measure the dc component of the optical power sent back by the filtered external cavity to the laser (measured to be $7 \mu \mathrm{W}$ ).

Fig. 4(a), taken with the laser resonant with the filter center, shows the measured optical power spectrum for very low 


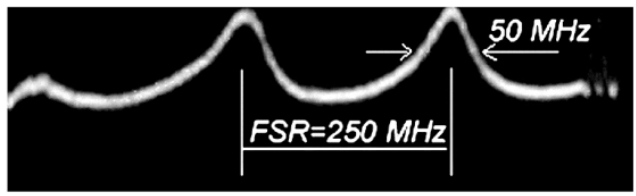

(a)

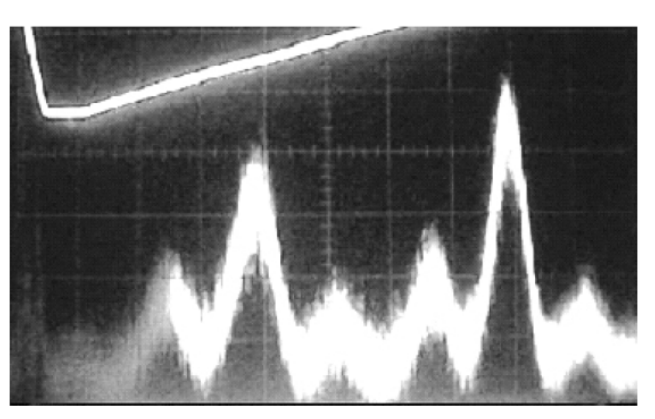

(c)

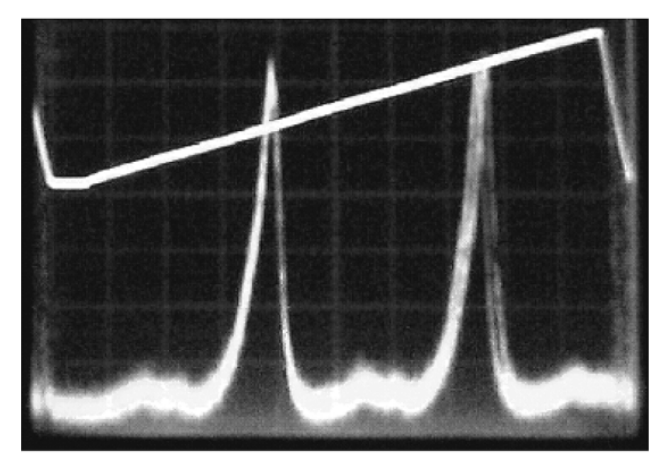

(b)

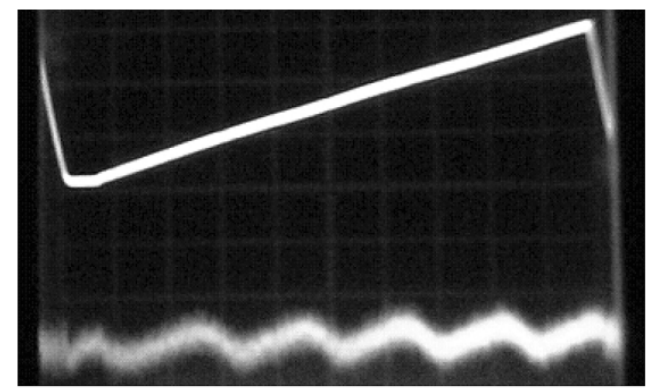

(d)

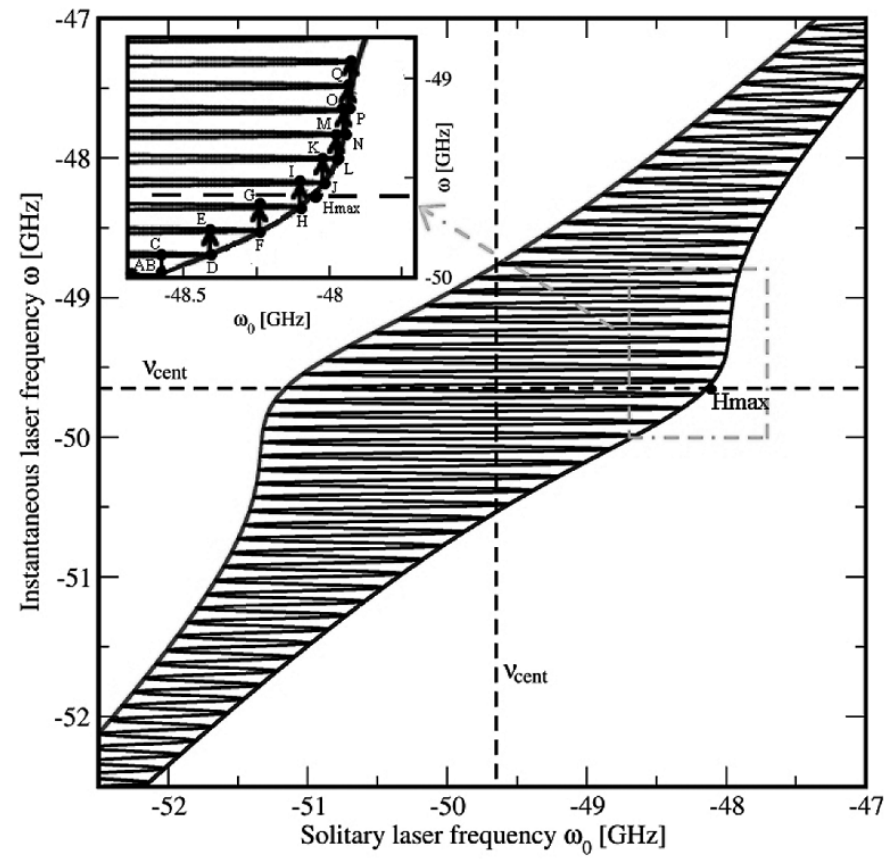

Fig. 3. Wide filter case. Optical spectra of the laser measured with a scanning FPI (top half of each panel shows the ramp applied to modulate the injection current to the solitary laser). The scanning range includes more than one FSR The filter width is $8 \mathrm{GHz}$. (a) Free-running laser spectrum, showing a linewidth of $50 \mathrm{MHz}$. (b) Relative feedback of $-32 \mathrm{~dB}(3 \mu \mathrm{W})$ leading to a narrowing of the laser linewidth. (c) Relative feedback of $-29 \mathrm{~dB}(7 \mu \mathrm{W})$ leading to undamping of the relaxation oscillations. (d) Relative feedback of $-25 \mathrm{~dB}(18 \mu \mathrm{W})$ showing coherence collapse in the laser. (e) Numerical simulation of the actual laser frequency as a function of the solitary laser frequency for wide filter case. Both frequencies are relative to threshold. Note that the peaks of the nearest ECMs, denoted by $H$ and $I$, do not coincide with the filter center frequency $\left(H_{\max }\right)$. Filter HWHM is $L=4 \mathrm{GHz}$. Relative feedback level is $-25 \mathrm{~dB}$.

feedback $((<-55 \mathrm{~dB})$ and it is similar to the solitary DL spectrum, exhibiting a $50-\mathrm{MHz}$ linewidth (the upper half of the figure shows the signal used for scanning the diagnostic Fabry-Perot inerferometer FPI as a function of time with an amplitude such that more than one FSR $=200 \mathrm{MHz}$ range is scanned). For a feedback power of $1.5 \mu \mathrm{W}(-34 \mathrm{~dB})$, the spectrum exhibits a single, narrow 18-MHz FWHM peak indicating a substantial line narrowing [Fig. 4(b)]. For the maximum obtainable feedback power $7 \mu \mathrm{W}(-29 \mathrm{~dB})$, the observed width of the main peak, $11 \mathrm{MHz}$, corresponds to the resolution of the FPI [Fig. 4(c)]. This indicates a further linewidth reduction. Two small satellite peaks on both side of the central peak are barely visible. The back-convolution in the $250-\mathrm{MHz}$ FSR FPI makes it difficult to estimate the exact frequency involved in this phenomenon, but it is reasonable to speculate that it is a multiple of the relaxation oscillation frequency. Comparison 


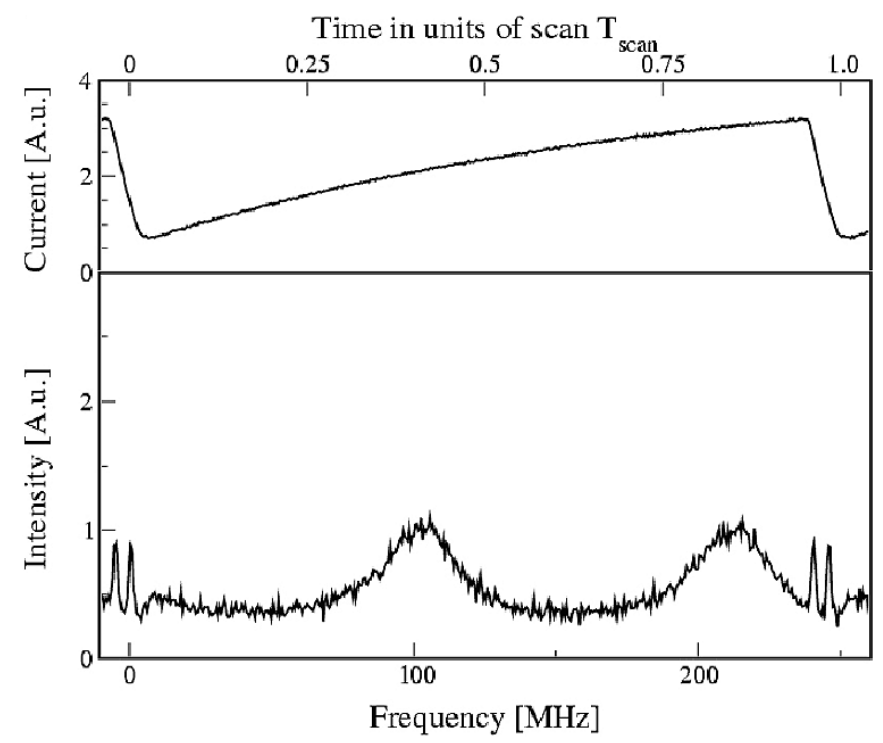

(a)

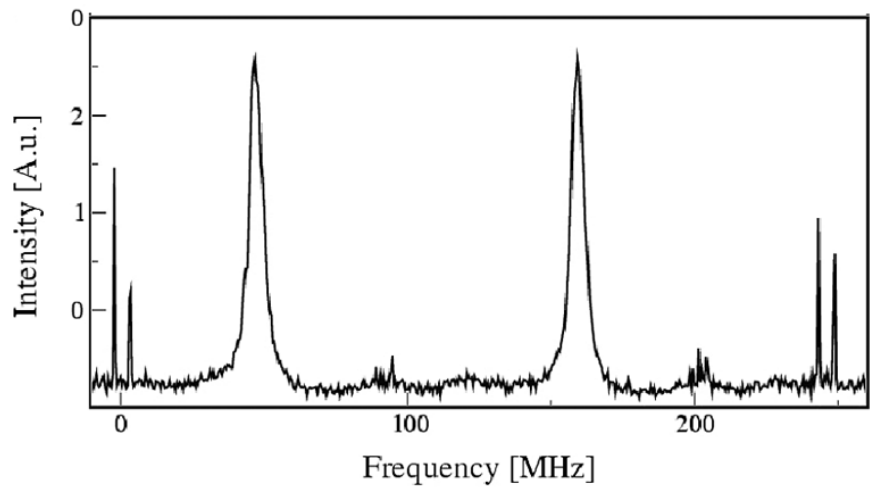

(c)

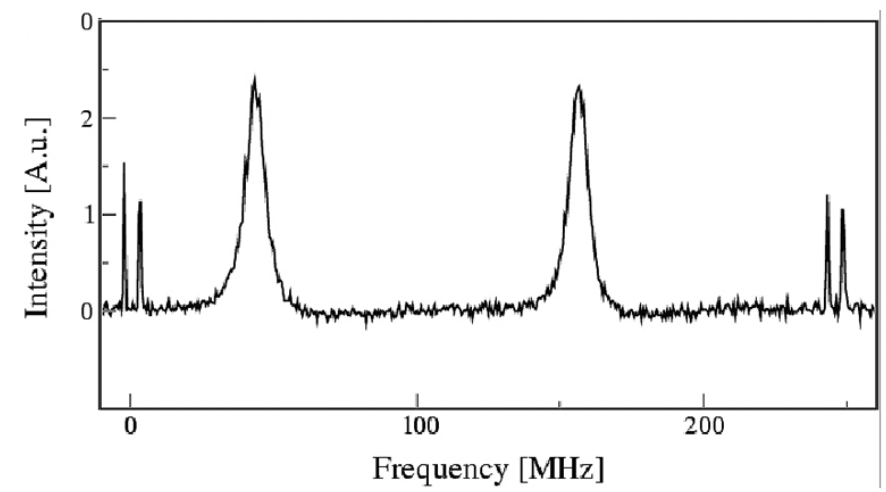

(b)

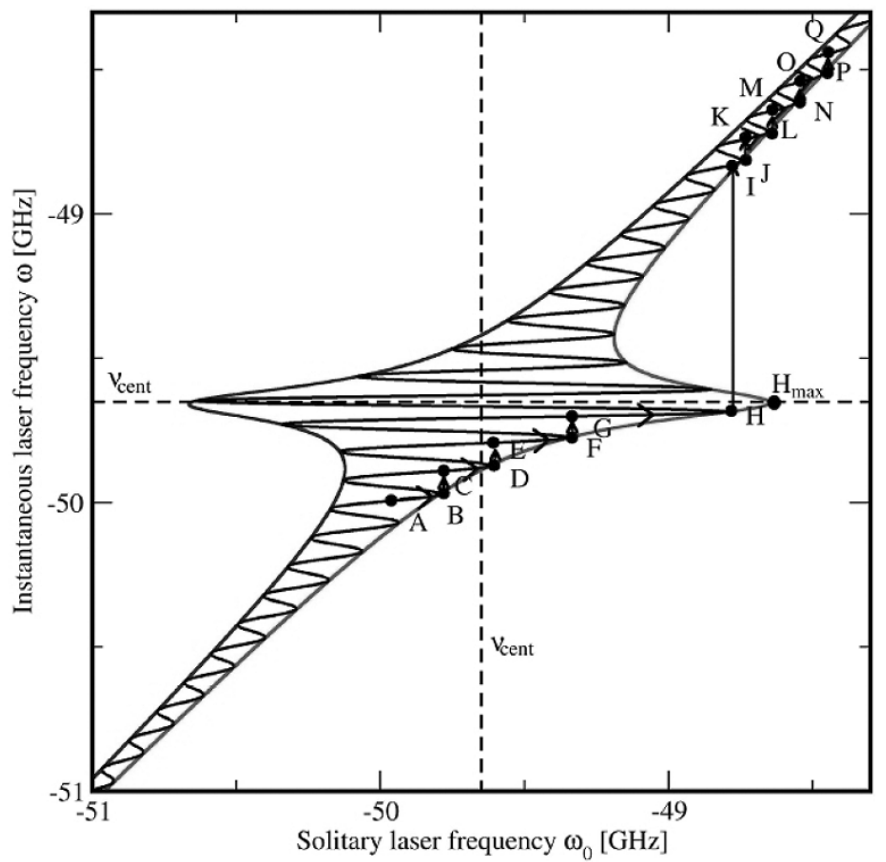

(d)

Fig. 4. Intermediate filter case. Optical spectra of the laser measured with a scanning FPI (top half of each panel shows the ramp applied to modulate the injection current to the solitary laser). The scanning range includes more than one FSR. The filter width is $700 \mathrm{MHz}$. (a) Free-running laser spectrum (feedback level below $-55 \mathrm{~dB}$ ), showing a linewidth of $50 \mathrm{MHz}$. (b) Relative feedback of $-34 \mathrm{~dB}(1,5 \mu \mathrm{W}$ ), leading to a narrowing of the laser linewidth. (c) Relative feedback of $-29 \mathrm{~dB}(7 \mu \mathrm{W})$ leading to a slight undamping of the relaxation oscillations. Numerical simulation of the actual laser frequency as a function of the solitary laser frequency for intermediate filter case. Both frequencies are relative to threshold. Filter FWHM is $700 \mathrm{MHz}$. ECM $=100 \mathrm{MHz}$. Relative feedback level is $-29 \mathrm{~dB}$.

with the Fig. 3(c) shows that the RO are much less undamped with the intermediate filter for the same amount of feedback.

Fig. 4(d) summarizes the simulated dynamics in the same way as in Fig. 3(e) and shows the frequency $\omega$ as a function of the solitary laser frequency $\omega_{0}$ (relative to threshold). The filter center frequency at $\omega_{0} \sim-49.65 \mathrm{GHz}$ is indicated. All the phenomena present in the wide filter case of Fig. 3(e), such as frequency jumps, bistability in frequency, and hysteresis, are seen in Fig. 4(d) as well [9]. When the pump current is decreased, the solitary laser frequency increases and the frequency of the external cavity laser moves along the oscillating snake contour in the same way as for wide-filter case.
We see from Fig. 4(d) that $H_{\max }$ and the top of the nearest external cavity mode do not lie at the same frequency. The main difference with respect to Fig. 3(e) lies in the point $H$ since this point is now located further away from the top $H_{\max }$ of the filter profile. From the measured data for an intermediate filter width, the ratio of the feedback powers at $H$ and $H_{\max }$ is $1 / 3$ whereas it was $9 / 10$ for the wide filter. It is this mismatch between the filter center frequency and the nearest ECM that results in a decrease in the maximum feedback power available. It is also clear that this mismatch increases as the filter narrows

While the results in Fig. 3(a)-(d) and Fig.4(a)-(c) are for the laser resonant with the center frequency of the filter, we next 


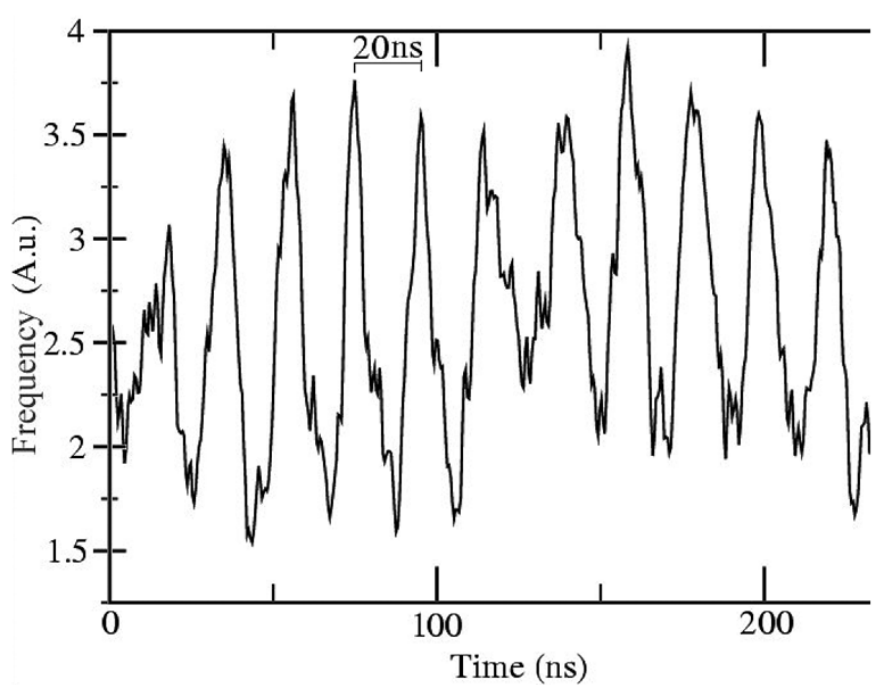

Fig. 5. Experimentally observed time series showing oscillations in the frequency of the laser when subject to filtered optical feedback. The period of the oscillations corresponds to an external delay of $6 \mathrm{~m}(\sim 20 \mathrm{~ns})$. The frequency excursions are about $1 \mathrm{GHz}$.

look at the temporal dynamics of the instantaneous optical frequency of laser light at a detuning of about $200 \mathrm{MHz}$ from the filter center frequency. Fig. 5 shows the signal detected on PDC after a frequency to power conversion by the diagnostic filter, and it is seen that the instantaneous laser frequency varies periodically with time with a period of $20 \mathrm{~ns}(50 \mathrm{MHz})$, which corresponds to the external cavity round trip length $L=3 \mathrm{~m}$. Note that the simultaneous power variations on PDB remain below the detection level. The period of the frequency modulation signal was also measured as a function of the external cavity length $L$ [13], which confirmed that the temporal dynamics of the laser frequency are directly proportional to the external cavity length $L$. Since there are no corresponding oscillations in the intensity of the light, it is clear that the frequency oscillations are not due to an undamping of the relaxation oscillations, but rather a feedback-delay-induced effect, and we refer to them as "external-delay-induced frequency oscillations" (EDFO). The origin of the frequency oscillations in terms of a dynamical picture has been recently reported, wherein we have also proved that a filter is essential for obtaining the periodic frequency variations without any accompanying power variations [13].

Next, we will investigate the conditions under which the EDFO occur. First of all we note that they do not occur for the wide filter case. Now, in order to investigate the dependence on laser frequency detuning with respect to the filter center frequency, the injection current is scanned while the feedback and the frequency of the laser output are monitored. Fig. 6 shows the result. In Fig. 6(a), the power transmitted through the filter is shown as a function of the solitary laser frequency, measured on PDE (bandwidth 0-300 KHz). In Fig. 6(b), recorded simultaneously, we show the ac component measured on PDC (bandwidth $300 \mathrm{KHz}-250 \mathrm{MHz}$ ). Also shown in Fig. 6 is the triangular pump current by which the solitary laser frequency is scanned. The dc component in Fig. 6(a) shows the average feedback power when the solitary laser frequency is scanned
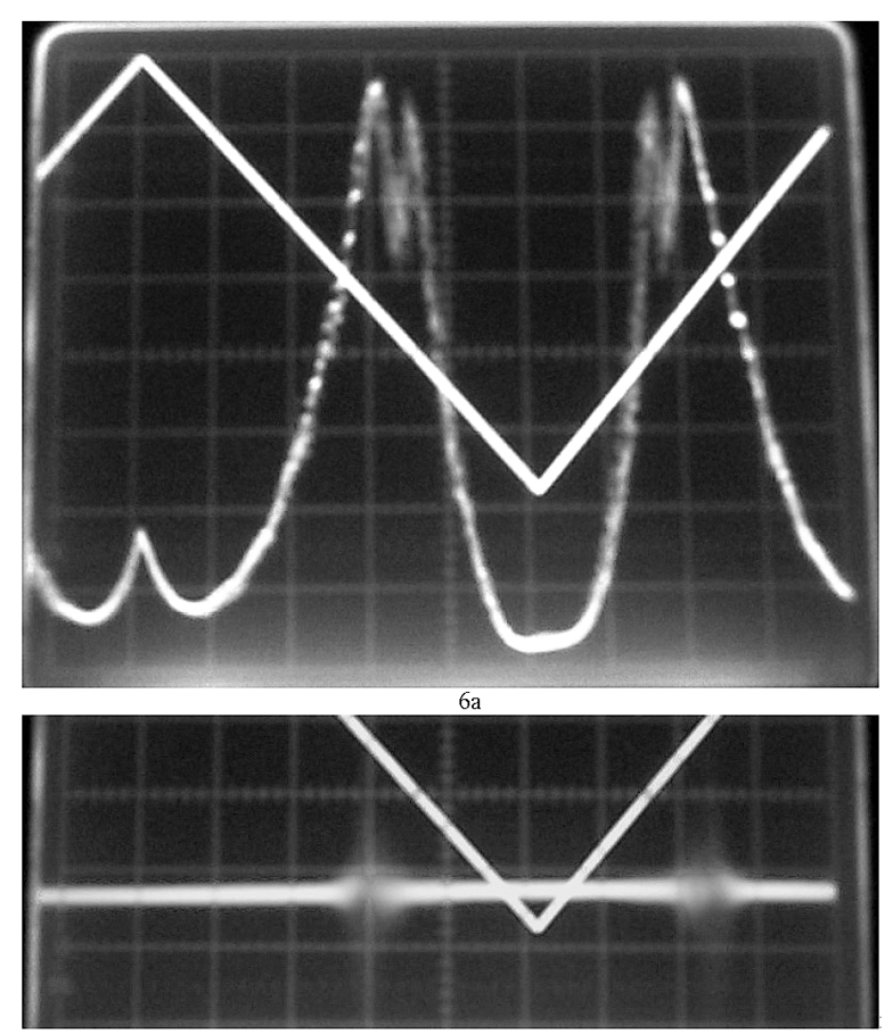

$6 b$

Fig. 6. (a) Scan of the pump current and the DC component of the light transmission through the filter, measured on PDE. The dotted structure on one flank of the filter transmission is a result of the laser locking to successive ECMs as the pump current is scanned. The "hole" in the vicinity of the peak is due to the mode mismatch effect discussed in the text. The blurred region near the peak indicates frequency dynamics faster than the detector response time. (b) AC component of the light transmitted through the filter. Figure also shows the pump current scan. The blurred region, which occurs at the same pump current as in Fig. 6(a), is due to an "external-delay-induced frequency oscillations" (EDFO) in the laser light. Note that except for regions where there are dynamics, the trace is flat, implying minimal intensity and frequency variations.

up and down relative to the filter. On the red side of the filter (left flank of the filter for decreasing pump current and right flank for increasing current) the filter profile exhibits dots that correspond to external cavity modes selected by the filter and to which the laser locks successively [8]-[10]. On the blue side, the transmitted power exhibits a "hole" in the vicinity of the top of the filter followed by a blurred curve. The hole is a manifestation of the blurred region that is due to EDFO dynamics that fall outside the detector bandwidth. This is also seen in Fig. 6(b) where the AC component of the instantaneous frequency is depicted. The trace of Fig. 6(b) is flat, except for the zones where the EDFO occurs. Note that Fig. 5 has been measured within these zones.

\section{Narrow Filter Case $\left(\delta \nu_{f} \ll \nu_{R O}\right.$ and $\left.\delta \nu_{f}<\nu_{\mathrm{ECM}}\right)$}

In this case, the external cavity length is set to $L=1.6 \mathrm{~m}$ corresponding to an external mode spacing of $95 \mathrm{MHz}$, and the distance between M1 and M2 was $d=11 \mathrm{~cm}$ with a finesse $f=8$. Thus, the filter free spectral range was $1.36 \mathrm{GHz}$ and its FWHM bandwidth was $\delta \nu_{f}=170 \mathrm{MHz}$, a value much smaller than the RO frequency. 


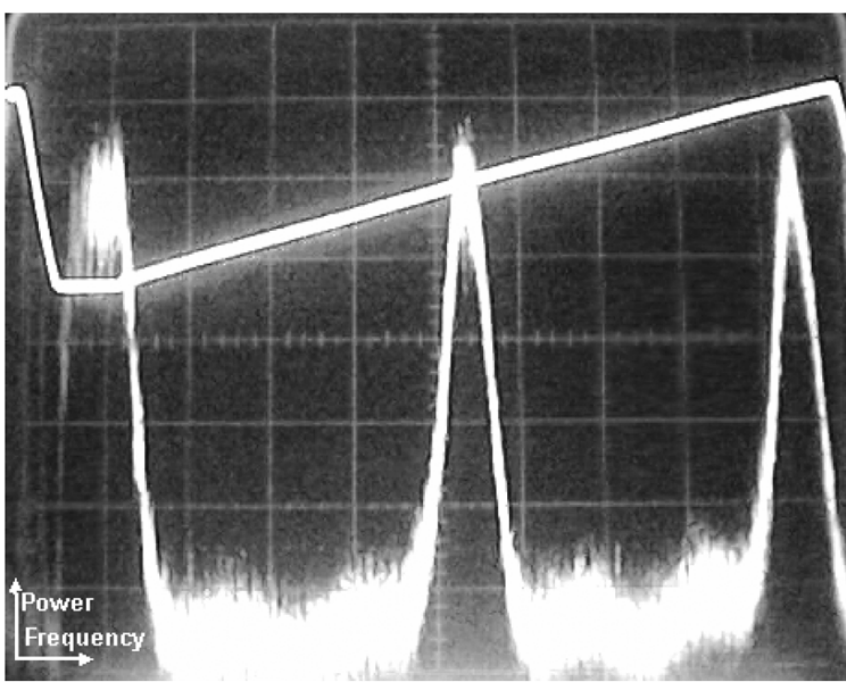

Fig. 7. Narrow filter case. Optical spectra of the laser measured with a scanning FPI. The filter width is $170 \mathrm{MHz}$. The spectrum, for maximum available relative feedback of $-32 \mathrm{~dB}(3 \mu \mathrm{W})$, shows a narrowing of the laser linewidth.

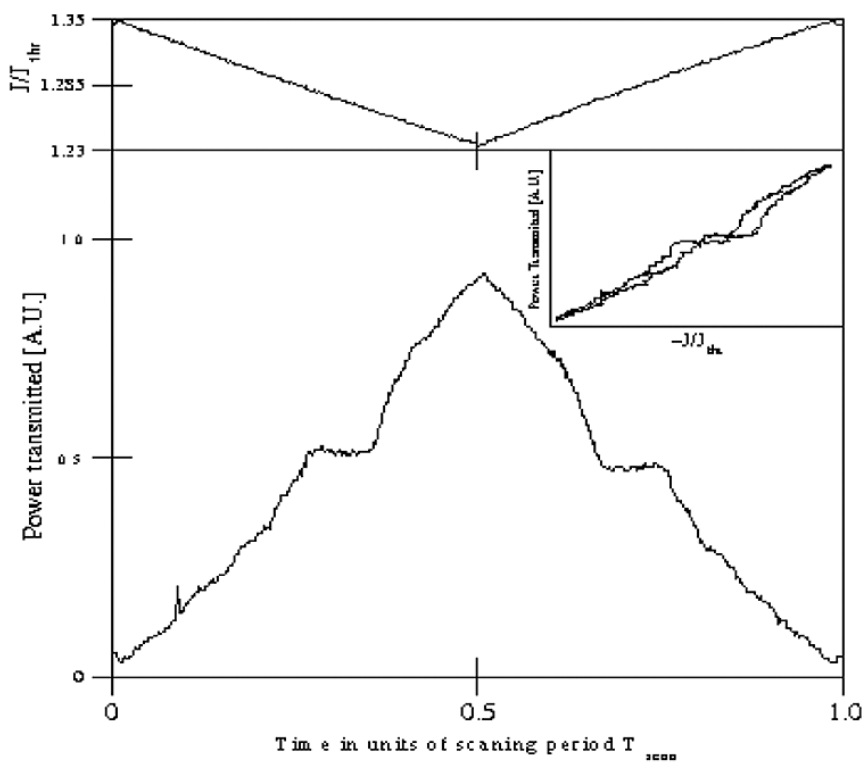

Fig. 8. Upper trace is the triangular ramp of the pump current. Lower trace shows the locking of the laser to an ECM. The filter width is $170 \mathrm{MHz}$. Inset is an $\mathrm{XY}$ plot of the power transmitted versus the injection current exhibiting frequency locking hysteresis and global bistability.

The optical spectrum measured with the FPI in arm A is shown in Fig. 7 for the maximum available feedback level of $3 \mu \mathrm{W}(-32 \mathrm{~dB})$. The solitary laser linewidth was $50 \mathrm{MHz}$. The pump current was $58.45 \mathrm{~mA}$. The laser linewidth was reduced to $\sim 25 \mathrm{MHz}$ indicating an improvement of the laser stability through filter assisted locking to an external-cavity mode. In Fig. 8, we show a measurement of the laser frequency as a function of the pump current that shows a locking to the external filter. The laser frequency is scanned with a triangular waveform superimposed on the bias current. The amplitude and the offset of the triangular waveform are chosen such that the pump current varies between 58 and $59 \mathrm{~mA}$, and such that the laser frequency range overlaps with one of the transmission peaks of

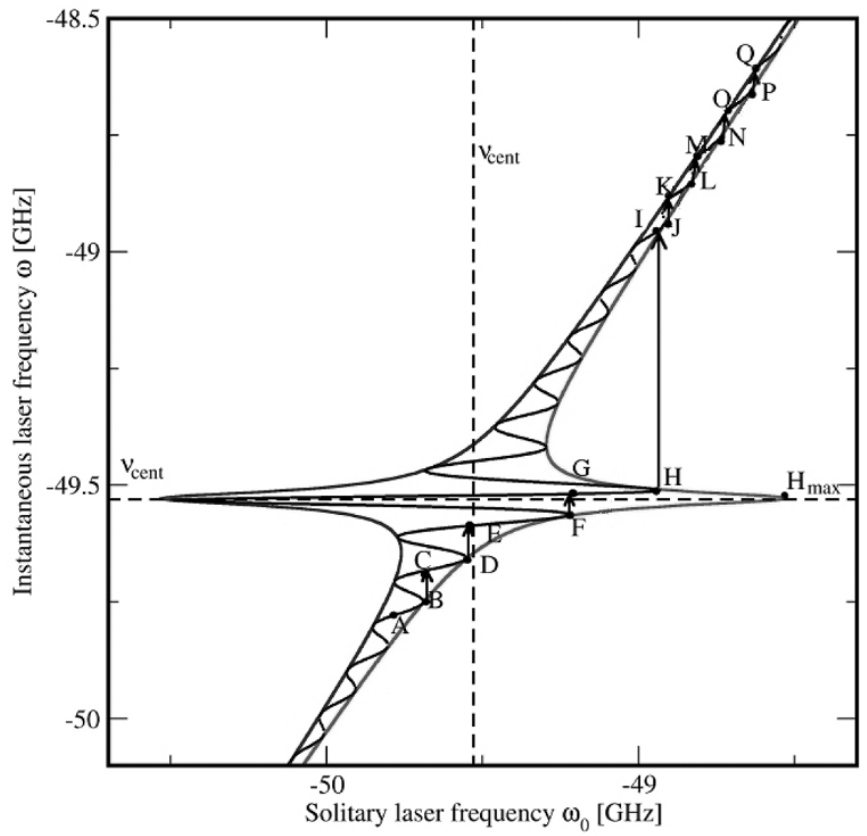

Fig. 9. Numerical simulation of the actual laser frequency as a function of the solitary laser frequency for narrow filter case. Both frequencies are relative to threshold. Filter FWHM is $170 \mathrm{MHz}$. ECM $=95 \mathrm{MHz}$. Relative feedback level is $-32 \mathrm{~dB}$.

the filter curve. The frequency of the laser system is shown as a function of time (lower trace), while the pump current is scanned down and up (upper trace). The frequency is shown to follow in first approximation the pump current except for two flat steps which are the ECM selected by the filter F, one for the increasing current, and one for the decreasing pump current. We note that there exist two frequency locking ranges, slightly different, separated by $90 \pm 10 \mathrm{MHz}$, i.e., corresponding to the ECM spacing for this case.

The corresponding simulations for a narrow filter are shown in Fig. 9 for the narrow filter. In the steady-state regime phenomena similar to the wide and intermediate filter cases, like frequency jumps, bistability, and hysteresis, and the global bistability, can be observed for narrow filter as well. However, it should be emphasized that the previously mentioned mode mismatch effect is more pronounced here. Indeed, the filter bandwidth being narrower, the mismatch between the filter central frequency and the external cavity modes may become proportionally more important. This may result in an even bigger difference between the power measured when the compound system operates in $\mathrm{H}$ and the maximum power measured at the top of the filter in the absence of feedback, $H_{\max }$ (Fig. 9). The $3 \mu \mathrm{W}$ maximum feedback power is smaller than the $7 \mu \mathrm{W}$ obtained for intermediate filter case. Thus, the mode mismatch effect becomes dominant as the filter width narrows.

\section{SUMMARY AND DISCUSSION}

A systematic study of the effect of the filter width on the dynamical response of a semiconductor laser under FOF reveals: 1) that the EDFOs occur for intermediate filter widths, and on a time scale that is determined by the delay time; 2 ) that the mode 
mismatch effect, which is observed in the form of reduced feedback power, becomes increasingly prominent with smaller filter widths; and 3) that the ability of the filter to extinguish the relaxation oscillations becomes stronger as the filter gets narrower.

We have discussed earlier, and in previous work, the origin of the EDFOs for intermediate filter widths [13]. They are directly related to the nonlinear frequency response of the filter. We emphasize that the effect is different from the nonlinear effect in power that is related to the $\chi^{(3)}$ properties of a material, or the nonlinear gain of a semiconductor material. Here, the frequency oscillations arises from a combination of the laser's frequency shift due to optical feedback and the nonlinear frequency dependence of the amount of light sent back by the filter to the laser. It is interesting to note that the EDFOs arise when two competing factors, the nonlinearity of the filter and its bandwidth, are appropriately balanced against each other. The ideal conditions for observing EDFOs is to have a filter that is narrow enough to suppress the relaxation oscillations, and yet wide enough to include several ECMs inside the filter profile. If the filter is too narrow, then one can clearly eliminate the relaxation oscillations, but then there may not be enough ECMs under the filter profile to produce the desired dynamics. On the other hand, a very wide filter will envelop several ECMs, but the relaxation oscillations will also leak into the dynamics. These competing parameters, therefore, offer useful tools for the control of the dynamics in a semiconductor laser with FOF and for design of all-optical systems.

The data presented in previous reports [8]-[10] on the steady-state properties of semiconductor lasers subject to FOF contained some hints of the mode mismatch effect noted in this paper. In our experiments, the effect became increasingly pronounced as the filter was narrowed. Specifically, in the absence of any filter, the feedback power available measured with a power meter was about $20 \mu \mathrm{W}$. However, when filters of $8 \mathrm{GHz}, 700$, and $170 \mathrm{MHz}$ were employed, the maximum feedback power available was measured to be 18,7 , and $3 \mu \mathrm{W}$, respectively. The phenomenon of mode mismatch is partly related to the ability of the filter to limit the intrusion of the relaxation oscillations. If the filter is narrower than the relaxation oscillation frequency, then a certain amount of light contained in those frequency components is no longer available for feedback since it is annihilated during the multipass interference process within the filter. However, this is not the complete answer because it only applies to situations in which the system shows dynamics. If filtering were the only mechanism contributing to a reduction in the feedback light, then one would find this "lost" power in the relaxation oscillation sidebands of the laser optical spectrum (on PDA), which we do not. In fact, only a part of the lost light is located outside the filter profile, which implies that there must be other mechanisms at play that cause a reduction in the available feedback power.

The other mechanisms arises as follows: as the filter center frequency moves through the ECM frequencies, it is quite likely that the ECM will not coincide with the center frequency of the filter, thereby reducing the maximum feedback power available. As the filter is made narrower, there are fewer ECM's within the filter profile, and the likelihood of an overlap between an ECM and the filter center frequency becomes smaller, and the mismatch be- tween a ECM and the filter frequency becomes larger. This mechanism will be most important in case of locking to an ECM and it is unique to coherent feedback, since it is not meaningful to talk of ECMs in case of incoherent feedback. In general, it is the combination of the filter's ability to remove the relaxation oscillations, and the mismatch between the ECM and filter frequency, that leads to the so called mode mismatch effect.

Finally, while our experiments utilized a filter that is narrower than the relaxation oscillations to remove these oscillations, one could conceivably, with the narrow filter, still induce those laser dynamics that are driven by the undamping of the relaxation oscillations. This could be done, for example, by arranging a multiple of the filter higher order transmission peak to coincide with the RO frequency, so that the feedback light that is modulated by the relaxation oscillation frequency is fed back to the laser. Another possibility is to simply detune the narrow filter from the laser by an amount corresponding to the relaxation oscillation frequency of the laser. Then, the relaxation oscillations would fall within the filter profile and be fed back into the laser. In both of these cases, one could excite dynamics that are dominated by the relaxation oscillations.

\section{CONCLUSION}

We have presented, both experimentally and with simulations, the influence of the filter width on the dynamics produces by filtered optical feedback in a semiconductor laser. The principal result is that one can excite nonlinear dynamical behaviors in the frequency of the light from the laser, and that these dynamics occur on a time scale that depends on the time delay of the feedback field. We have compared the effect of three types of filters, a wide filter, an intermediate filter and a narrow filter, and shown that the filter width can be used to exercise control over the dynamics of the laser. We demonstrated that through an appropriate choice of the filter width one can suppress the effect of the relaxation oscillations. Lastly, we have discussed the mode mismatch effect, which arises from a frequency detuning between the filter center frequency and the closest external cavity mode, and will arise only for coherent feedback. Mode mismatch, in combination with the filter's ability to suppress frequency components outside its bandwidth, can lead to a reduction in the maximum available feedback power, which then prevents the undamping of the relaxation oscillations and coherence collapse. Potential applications deal with optical telecommunication, all-optical signal processing,chaos control for secure communication control, and design of all optical digital gates.

\section{REFERENCES}

[1] D. Lenstra and B. Krauskopf, Eds., Nonlinear Dynamics in Semiconductor Lasers. New York: Plenum, 2000.

[2] S. M. Verduyn-Lunel and B. Krauskopf, Nonlinear Dynamics in Semiconductor Lasers, D. Lenstra, Ed. New York: Plenum, 2000, pp. 66-86.

[3] G. H. M. van Tartwijk and D. Lenstra, "Semiconductor lasers with optical injection and feedback," Quantum Semiclass. Opt., vol. 7, pp. 87-143, 1995.

[4] E. A. Viktorov and P. Mandel, "Multimode semiconductor laser with selective optical feedback," Opt. Lett., vol. 25, pp. 1573-1575, 2000. 
[5] T. Heil, I. Fischer, W. Elsäßer, and A. Gavrielides, "Dynamics of semiconductor lasers subject to delayed optical feedback: The short cavity regime," Phys. Rev. Lett., vol. 87, pp. 243 901-243 9014, 2001.

[6] C. Masoller, "Noise-induced resonance in delayed feedback systems," Phys. Rev. Lett., vol. 88, pp. 034 102-034 105, 2002.

[7] "Feature Section on Optical Chaos and Applications to Cryptography," IEEE J. Quantum Electron., vol. 38, pp. 1138-1184, 2002.

[8] O. K. Andersen, A. P. A. Fischer, I. C. Lane, E. M. Louvergneaux, S Stolte, and D. Lenstra, "Experimental stability diagram of a diode laser subject to weak phase-conjugate feedback from a rubidium vapor cell," IEEE. J. Quantum Electron, vol. 35, pp. 577-582, Apr. 1999.

[9] A. P. A. Fischer, O. K. Andersen, M. Yousefi, S. Stolte, and D. Lenstra, "Experimental and theoretical study of filtered optical feedback in a semiconductor laser," IEEE J. Quantum Electron, vol. 36, pp. 375-384, Mar. 2000.

[10] T. Erneux, M. Yousefi, and D. Lenstra, "The injection laser limit of lasers subject to filtered optical feedback," in Proc. European Quantum Electronics Conf., Munich, Germany, 2003, Europhysics Conf. Abstracts 27E, EA3 04 THU.

[11] F. Rogister, P. Mégret, O. Deparis, M. Blondel, and T. Erneux, "Suppression of low frequency fluctuations and stabilization of a semiconductor laser subjected to optical feedback from a double cavity: Theoretical results," Opt. Lett., vol. 24, pp. 1218-1220, 1999.

[12] D. W. Sukow, M. C. Hegg, J. L. Wright, and A. Gavrielides, "Mixed external cavity mode dynamics in a semiconductor laser," Opt. Lett., vol. 27, pp. 827-829, 2002.

[13] A. P. A. Fischer, M. Yousefi, D. Lenstra, M. W. Carter, and G. Vemuri, "Experimental and theoretical study of semiconductor laser dynamics due to filtered optical feedback," Phys. Rev. Lett., vol. 92, pp. 023 901-023 904, 2004.

[14] A. Fischer, J. P. Goedgebuer, and H. Porte, "High-density wavelength switching obtained from a tunable multisectionlaser diode," Opt. Lett., vol. 24, pp. 765-767, 1999.

[15] G. Huyet, S. Balle, M. Giudici, C. Green, G. Giacomelli, and J. R. Tredicce, "Low Frequency fluctuations and multimode operation of a semiconductor laser with optical feedback," Opt. Commun., vol. 149, pp. 341-347, 1998

[16] M. Giudici, L. Giuggioli, C. Green, and J. R. Tredicce, "Dynamical behavior of semiconductor lasers with frequency selective optical feedback," Chaos, Solitons, Fractals, vol. 10, pp. 811-817, 1999.

[17] J. P. Goedgebuer, L. Larger, and H. Porte, "Optical cryptosystem based on synchronization of hyperchaos generated by a delayed feedback tunable laser diode," Phys. Rev. Lett., vol. 80, pp. 2249-2252, 1998.

[18] J. P. Goedgebuer, P. Levy, L. Larger, C.-C. Chen, and W. T. Rhodes, "Optical communication with synchronized hyperchaos generated electrooptically," IEEE J. Quantum Electron, vol. 38, pp. 1178-1183, July 2002.

[19] M. Yousefi, D. Lenstra, G. Vemuri, and A. Fischer, "Control of nonlinear dynamics of a semiconductor laser with filtered optical feedback," IEE Proc.-Optoelectron., vol. 148, pp. 223-237, 2001.

A. P. A. Fischer, photograph and biography not available at the time of publication.

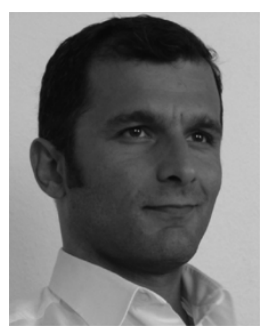

Mirvais Yousefi (S'00-M'03) was born in Kabul, Afghanistan, in 1975. He received the M.Sc. degree from the University of Lund, Lund, Sweden, in 1998, and the Ph.D. degree from the Vrije Universiteit, Amsterdam, The Netherlands, in 2003. The focus of his research was on the analysis of the dynamics of coupled semiconductor lasers in several configurations, such as filtered feedback and laterally coupled semiconductor lasers.

$\mathrm{He}$ is currently working on the fabrication of laterally coupled semiconductor lasers for chaotic encryption at the Opto-Electronic Device Group, Technical University of Eindhoven, Eindhoven, The Netherlands. His recent activities include the influence of noise on semiconductor laser dynamics, synchronization of semiconductor lasers for chaotic encryption purposes, and analysis of the dynamics of semiconductor lasers with filtered feedback. His research interests include semiconductor lasers, their dynamics, and the application of these dynamics.

Dr. Yousefi received the IEEE Lasers and Electro-Optics Society Graduate Student Fellowship in 2001.

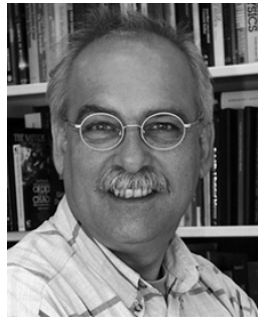

Daan Lenstra was born in Amsterdam, The Netherlands, in 1947. He received the M.Sc. degree in theoretical physics from the University of Groningen, Groningen, The Netherlands, and the Ph.D. degree from Delft University of Technology, Delft, The Netherlands. His thesis work was on polarization effects in gas lasers.

Since 1979, he researched topics in quantum electronics, laser physics, and condensed matter physics. In 1991, he joined the Vrije Universiteit, Amsterdam, holding a chair in theoretical quantum electronics. Since 2000, he has also been with COBRA Research Top Institute, Eindhoven University of Technology, Eindhoven, The Netherlands, where he occupies a chair in ultrafast photonics. His research interests include nonlinear and ultrafast dynamics of semiconductor optical amplifiers and diode lasers, quantum optics in small semiconductor structures, and near-field optics. He has (co)authored more than 210 publications in international scientific journals and (co)edited six books and several special journal issues. He acts regularly as program (co)chair for international meetings.

Dr. Lenstra is a member of IEEE Lasers and Electro-Optical Society, the Optical Society of America, and the European Physical Society.

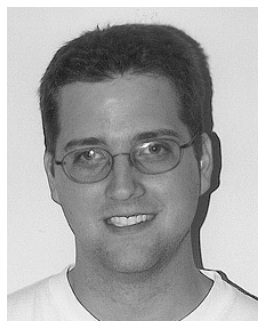

Michael W. Carter received the B.A. degree in physics from Hanover College, Hanover, IN, in 1994, and the M.S. degree in physics in 2001 from Indiana University Purdue University Indianapolis (IUPUI), Indianapolis, where he is currently working toward the $\mathrm{Ph} . \mathrm{D}$. degree in physics.

His research interests includes dynamics in diode lasers subject to optical feedback as well as the study of pulse propagation in resonant, three level systems.

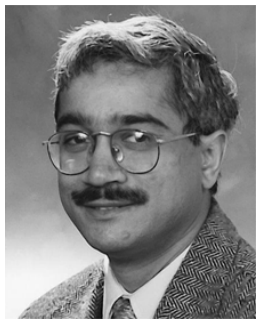

Gautam Vemuri received the B.Sc. degree (with honors) in physics from St. Stephen's College, University of Delhi, Delhi, India, in 1984, the Sc.M. degree in physics from Brown University, Providence, RI, in 1990, and the Ph.D. degree in physics from the Georgia Institute of Technology, Atlanta, in 1990.

In 1992, after a Postdoctoral stint with JILA, Boulder, $\mathrm{CO}$, he joined Indiana University-Purdue University Indianapolis (IUPUI), Indianapolis, IN, as an Assistant Professor. He is currently a Professor of Physics, and the Chair of the Department of Physics, IUPUI. His research interests include the general area of nonlinear dynamics of semiconductor lasers with emphasis on feedback and time-delayed induced dynamics. He also has a strong interest and expertise in stochastic properties of lasers and in laser noise-induced effects in laser-atom interactions.

Dr. Vemuri was awarded the University Gold Medal for securing first rank in the B.Sc. (honors) physics program. 